\title{
Effects of non-homogeneity and oxide coating on silver nanowire networks under electrical stress: comparison between experiment and modeling.
}

\author{
Dorina T. Papanastasiou ${ }^{1}$, Nicolas Charvin ${ }^{2}$, Joao Resende ${ }^{3}$, Viet Huong Nguyen ${ }^{4}$, \\ Abderrahime Sekkat ${ }^{1}$, David Muñoz-Rojas ${ }^{1}$, Carmen Jiménez ${ }^{1}$, Lionel Flandin ${ }^{2}$, \\ Daniel Bellet ${ }^{1, *}$ \\ ${ }^{1}$ Univ. Grenoble Alpes, CNRS, Grenoble INP, LMGP, F-38000 Grenoble, France \\ ${ }^{2}$ Univ. Grenoble Alpes, Univ. Savoie Mont Blanc, CNRS, Grenoble INP, LEPMI, 38000 Grenoble, France \\ ${ }^{3}$ AlmaScience Colab, Madan Parque, 2829-516 Caparica, Portugal \\ ${ }^{4}$ Faculty of Materials Science and Engineering, Phenikaa University, Hanoi 12116, Vietnam
}

E-mail: daniel.bellet@ grenoble-inp.fr

Received xxxxxx

Accepted for publication $\mathrm{xxxxxx}$

Published xxxxxx

\begin{abstract}
Silver nanowire (AgNW) networks are among the most promising indium-free, flexible transparent electrodes for energy, lighting and heating devices. However, the lack of stability of such networks is a key factor that limits their industrial application. While applications require homogeneous networks, non-homogeneous AgNW networks are intentionally prepared in the present work to probe the mechanisms leading to failure under electrical stress. We show that induced non-homogeneities have a strong impact both on the spatial distribution of temperature (measured by IR imaging) and the current density throughout the electrode (as deduced from modeling). Regions with higher current density under elevated electrical stress are correlated to the origin of degradation. Furthermore, the influence of a zinc oxide $(\mathrm{ZnO})$ layer on electrical performances of non-homogeneous specimens is studied. Thanks to $\mathrm{ZnO}$ coating, the tortuosity of electrical potential lines measured by the one-probe mapping technique is much lower than for bare networks. Additionally, coated network electrical failure occurs at $40 \%$ higher voltage compared to bare network, over $18 \mathrm{~V}$, while reaching superior power-induced heating of $360{ }^{\circ} \mathrm{C}$. The results presented here will contribute to the design and fabrication of more robust nanowire networks, particularly for application in transparent heaters.
\end{abstract}

Keywords: transparent electrodes, simulations, nanocomposite, IR imaging, Atmospheric-Pressure Spatial Atomic Layer Deposition

\section{Introduction}

Transparent conductive materials (TCMs) are widely used in various applications, including OLEDs, touchscreens, solar cells, smart windows, transparent heaters, and more.[1-3] The most common TCMs used over the last decades have been transparent conductive oxides (TCOs), mainly indium tin oxide (ITO), due to their outstanding optical and electrical properties. However, ITO suffers from well-identified drawbacks, such as scarcity of indium and brittleness. [4-7] The increasing demand for flexible materials with excellent 
optical and electrical properties have prompted investigations on alternative solutions.[8-10] Several candidates have been extensively studied over the last decade, namely, carbon nanotubes (CNTs),[11] graphene,[12] conductive polymers,[13] metallic grids[14], and metallic nanowires (MNWs),[15-17]. Numerous studies on silver nanowire (AgNW) networks have shown their outstanding properties.[18,19] For example, randomly deposited AgNW networks present state-of-the-art sheet resistance values below $10 \Omega /$ sq and optical transparency of $90 \%$, containing a small amount of silver $\left(50-150 \mathrm{mg} / \mathrm{m}^{2}\right)$ and prepared by low-cost and scalable fabrication techniques.[20-23] Recent studies report interesting methods to improve their response to mechanical stresses thanks to the sintering between the nanowires and their adhesion with the intermediate layers and substrates.[24-26] These improvements/enhancements are essential for next generation of optoelectronic devices. AgNW networks are thus ideal candidates for flexible and stretchable applications, since they may be bent or twisted without losing their electrical and optical properties, as opposed to ITO and many other TCMs.[27] Thanks to their advanced properties, AgNW networks have been investigated for integration in many devices, such as flexible solar cells, [28,29] OLEDs,[30] displays,[31] transparent heaters,[32,33] radio-frequency antennas,[34] electromagnetic interference shielding,[35,36] thin film transistors,[37] flexible supercapacitors,[38] sensors, [39] or smart windows.[40]

However, one of the main limitations for the application of AgNW networks in devices is their lack of stability under electrical, thermal, mechanical, and chemical stresses. [41-46] The degradation mechanisms have been studied with different approaches, from the individual nanowire or the junction between two nanowires, up to the macroscopic failure of the whole network.[47-51] Sannicolo et al. used in situ experiments to show that the failure dynamics of AgNW networks under electrical stress occur through a localized mechanism.[52] In particular, the formation of electrical resistive hotspots could be clearly visualized by IR imaging of the surface temperature of the network during a voltage ramp. The breakdown consists of two steps: the creation of a crack, thermally induced by a localized high current density, followed by its propagation nearly parallel to the equipotential lines. Such response has been reported also recently by Zhu et al.[53] Therefore, for the successful integration of AgNW network into devices, it is crucial to improve their thermal and electrical stability. In principle, this can be achieved by preventing the surface diffusion of silver atoms that leads to morphological instabilities of the nanowires. Several studies have shown a stability enhancement by coating the networks with thin metal oxide layers, such as zinc oxide $(\mathrm{ZnO}),[54,56,57] \quad$ aluminium oxide $\left(\mathrm{Al}_{2} \mathrm{O}_{3}\right),[58-61]$ aluminium-doped zinc oxide (AZO),[62-64], or with graphene oxide (GO).[55,65] Despite the brittleness of metal oxide coatings, the transparent electrodes based on MNW/metal oxide nanocomposites still exhibit high mechanical stability, mainly attributed to the excellent flexibility of AgNW networks, when associated to the very thin layer of the oxide coating. MNW coated with a thin layer of oxide also present an enhanced stability when such networks are submitted to electrical stress, as shown by Khan et al. and by Nguyen et al. with $\mathrm{ZnO}$ and $\mathrm{AZO}$, respectively.[54,64] In addition to stability enhancement, these oxides are also of clear interest for many applications. [66] Ag-based core-shell nanowires fabricated by sol-gel is another promising way to achieve transparent electrodes with enhanced stability.[67,68]

In spite of many experimental efforts devoted to the stability improvement of AgNW-based TCMs, a deep understanding of the main mechanisms leading to network failure is still missing. As experimentally observed, the degradation of the AgNW networks usually initiates with a local defect that subsequently propagates over a larger area. In other words, the discrete nature of the network at a very local scale seems to result in flaws, while the homogeneity of the structure controls the propagation. So far, the great majority of investigations have focused on homogeneous AgNW networks, but actual samples tend to present nonhomogeneities, such as the so-called "coffee rings", or localized areas where the density of nanowires is much lower or higher than the average network density.[15,69] Such intrinsically heterogeneous nature of the network may then regulate the degradation rate under electrical stress.[42] To evaluate the impacts of such non-homogeneities on the network stability and failure mechanisms, non-homogeneous AgNW networks were intentionally designed and fabricated in this work, by using a shadow mask during spray deposition. Therefore, we studied the spatial distribution of the electrical potential and surface temperature, both experimentally and through simulations. The effect of a thin $\mathrm{ZnO}$ coating on the stability of the AgNW non-homogeneous networks was as well evaluated. To our knowledge, this has not been investigated previously and appears critical for the endorsement of AgNW networks in actual modern devices. Atmospheric pressure spatial atomic layer deposition (APSALD) has been chosen for the deposition of $\mathrm{ZnO}$ thin films as a low-cost, fast, vacuum-free, and scalable process.[70,71] Our results show that non-homogeneities are strongly correlated to the origin of failure due to the development of hotspots, and that oxide coatings homogenize the current distribution along the network even for non-homogeneous samples, rendering the networks more stable. 


\section{Experimental Methods}

\section{AgNW synthesis and networks fabrication}

AgNWs with an aspect ratio close to 100 (average diameter of $70 \pm 10 \mathrm{~nm}$ and an average length of $8 \pm 3 \mu \mathrm{m}$ ) were kindly provided from the research team of Jean-Pierre Simonato from CEA-LITEN in Grenoble, France, being produced as detailed in Mayousse et al.[72] The AgNWs were dispersed in methanol with an initial concentration of $3.1 \mathrm{~g} / \mathrm{kg}$ and diluted afterwards 30 times to obtain a concentration of $0.1 \mathrm{~g} / \mathrm{L}$. The substrates used, Alkaline earth boroaluminosilicate glass (Corning 1737, thickness $1.1 \mathrm{~mm}$ ), were cut in squares of $25 \times 25 \mathrm{~mm}^{2}$, rinsed with acetone, sonicated for $15 \mathrm{~min}$ in isopropanol, rinsed with distilled water, and finally dried with $\mathrm{N}_{2}$ gas. Spray deposition with a homemade airbrush (Harder \& Steenbeck) set-up was performed with a hot plate set to 110 ${ }^{\circ} \mathrm{C}$ and a nitrogen inlet with a 1.4 bar pressure. Bare AgNW networks exhibit a resistance of $20 \Omega / \mathrm{sq}$ and $90 \%$ optical transparency. Non-homogeneous specimens were fabricated using masks with triangular shapes, as shown in Figure $1 \mathrm{~b}$. These triangular areas were either free of $\mathrm{AgNWs}$ or presenting a higher areal mass density (amd) value (134 $\left.\mathrm{mg} / \mathrm{m}^{2}\right)$, compared to the rest of the network $\left(114 \mathrm{mg} / \mathrm{m}^{2}\right)$. Two types of masks were printed using a 3D printer with hightemperature resin (version FLHTAM01 by Formlabs, Inc.). After the AgNW networks fabrication, silver paste-based contacts were manually deposited at two opposite sides of the specimen.

\section{Oxide coating}

$\mathrm{ZnO}$ thin films were deposited with the AP-SALD homemade system of LMGP.[70,71] Diethylzinc (DEZ, (C2H5)2Zn, Aldrich), and water vapor were used as precursors for zinc, and oxygen, respectively. The substrate temperature was maintained at $200{ }^{\circ} \mathrm{C}$. The gas injector was fixed at $150 \mu \mathrm{m}$ from the substrate, which oscillated at $10 \mathrm{~cm} / \mathrm{s}$ between different precursor channels to produce 100 ALD cycles, corresponding to $30 \pm 5 \mathrm{~nm}$ thick $\mathrm{ZnO}$.

\section{Experimental characterizations}

AgNWs and film surface morphology were analyzed by field emission gun-scanning electron microscopy (FEG-SEM Environmental FEI QUANTA 250 and SEM ZEISS-Gemini 300) and transmission electron microscopy (JEOL JEM-2010 microscope). ImageJ software was used to calculate the amd (expressed in $\mathrm{mg} / \mathrm{m}^{2}$ ) of the AgNW networks by a wire detection plug-in (Ridge detection), estimating the geometrical density in SEM images, following the protocol described somewhere else.[64] Optical properties were analyzed using a Lambda 950 spectrophotometer from Perkin Elmer in the $250-2500 \mathrm{~nm}$ range. The electrical properties were investigated by experimental set-ups that allow in situ measurements. One-probe mapping, as previously described in details elsewhere,[52] was used to obtain the electrical potential distribution of the AgNW network. As shown schematically in Figure 1e, the left contact of the measured specimen was connected to the ground, while a $1 \mathrm{~V}$ bias was applied to the right one. The probe was sequentially positioned on a regular grid over the AgNW network, monitored by LabVIEW software, and each time its tip touches the network, the local electric potential is measured. The grid step size was set to $1 \mathrm{~mm}$. A classical 2-probe set-up in the edge of the specimen, using metallic forceps was used to apply the voltage by the mean of a Keithley 2602 source-measure-unit (SMU) during the voltage ramps. In parallel, the surface temperature spatial distribution of the specimens during the voltage ramp was measured by a FLIR A320G IR camera. The contact resistance between the probe and the AgNW network was measured using a silver paste layer as a specimen and its value was found less than $0.2 \mathrm{ohm}$. Therefore the contact resistance between the probe and the AgNW network can be neglected.

\section{Modeling}

Physical simulations of both homogeneous and nonhomogeneous AgNW networks were performed using Comsol Multiphysics ${ }^{\circledR}$ version 5.2 and the AC/DC Module. The software is based on finite element analysis and the physics of "Electric Currents, Shell" and "Heat transfer in solids" were used, coupling both electrical and thermal models and thus calculating the spatial distributions of the electric potential, current density, and surface temperature. Homogeneous thin Ag films on top of Corning glass were simulated to represent the AgNW networks. Although AgNW constitute nanomaterials for which sizes can be of the same order of magnitude of phonon mean free path, we considered for the sake of simplicity through Comsol simulations heat transfer modeling within a continuous medium. In the case of empty triangles, the associated sheet resistance is infinite within the triangles, while for the denser triangles the sheet resistance was set to $20 \Omega / \mathrm{sq}$, while the rest of the specimen was set to $25 \Omega / \mathrm{sq}$. The size of the simulated specimens was the same as the experimentally fabricated ones $\left(25 \times 25 \mathrm{~mm}^{2}\right)$ as well as the glass substrate size $\left(25 \times 25 \times 1.1 \mathrm{~mm}^{3}\right)$. A work plane was added, where rectangular contacts with a width of $1 \mathrm{~mm}$ were added on the left and the right sides with the appropriate electric boundary conditions.

In order to perform thermal simulations, three main mechanisms of heat losses must be taken into account: convection, conduction, and radiation. Considering the temperature is uniform across the sample and neglecting the conduction losses to external parts of the system, one can write the energy balance:[73]

$$
\begin{gathered}
I^{2} R=\left(m_{1} C_{1}+m_{2} C_{2}\right) \frac{d T(t)}{d t}+A\left(h_{1}+h_{2}\right)\left(T(t)-T_{0}\right)+ \\
\sigma A\left(\varepsilon_{1}+\varepsilon_{2}\right)\left(T(t)^{4}-T_{0}^{4}\right)(1)
\end{gathered}
$$


where the subscripts 1 and 2 denote respectively silver thin film and the substrate, $I$ is the applied electric current, $R$ is the resistance of the film, $A$ is the substrate area, $\sigma$ is the Stefan Boltzmann constant, $m$ is the mass, $C$ is the specific heat capacity, $h$ is the convective heat transfer coefficient, and $\varepsilon$ is the emissivity of the sides of the samples. $T(t)$ denotes the instant temperature of the sample, and the ambient temperature $T_{0}$ used in the model is $20^{\circ} \mathrm{C}$. From equation (1) one can see that the heat generated by the Joule effect (the left term) is dissipated through the temperature elevation in the conductive film and the substrate (the first term on the right side), the heat losses by convection (the second term) and radiation (the third term). In the model, the two layers are not physically separated so the conduction between them is considered ideal. In order to mimic as best as possible the experimental conditions, any external conduction losses are neglected. As shown in Figure S1a, during the experiments, the samples were not in direct contact with any other material since they were hold in air by the crocodile jaws connected to SMU. Thus, they present minimum conduction heat losses only through the electrical wiring. Regarding the later, an example of supplementary simulations presented in Figure $\mathrm{S} 1 \mathrm{~b}$, shows no influence of extra wiring to the values and distribution of surface temperature. To consider the radiation losses, the emissivity of both Corning glass and Ag are considered. To consider the convection losses, the following values of convective heat transfer coefficient have been reported in the literature for nanostructured or metallic films: $h=8-92 \mathrm{~W} /\left(\mathrm{m}^{2} . \mathrm{K}\right)$.[73] In the present work $h=10 \mathrm{~W} /\left(\mathrm{m}^{2} . \mathrm{K}\right)$ was applied for the glass substrate and $h=50 \mathrm{~W} /\left(\mathrm{m}^{2} . \mathrm{K}\right)$ for the silver layer $h=40 \mathrm{~W} /\left(\mathrm{m}^{2} . \mathrm{K}\right)$ for the less dense/conductive part in the middle of the denser triangles non-homogeneous case). More details of each material properties such as electrical and thermal conductivity, emissivity, and heat transfer coefficients are listed in Table $\mathrm{S} 2$ reported in Supplementary Information.

\section{Results and Discussion}

Three types of specimen with dissimilar structures have been used throughout the entire study as presented schematically in Figure 1a: i) a standard homogenous network as a reference, referred to as HOM, ii) a series of samples with two triangles without nanowires, referred to as ET for empty triangles, and iii) the same geometry but with the triangles containing a higher density of nanowires, referred to DT for dense triangles. Outside the triangles, all samples had the same density. To fabricate the non-homogeneous ET and DT samples, we used 3D-printed masks, shown in Figure 1b. Photos of the three different types of samples are presented in Figure $1 \mathrm{~b}$ as well. For the sake of reproducibility, during each AgNW spray deposition, the resistance was measured in situ with a resistance monitor, as presented in a previous work; [64] this enabled to better control the actual $\mathrm{AgNW}$ density.
Further experimental details about the deposition process can be found in the Experimental Methods section.
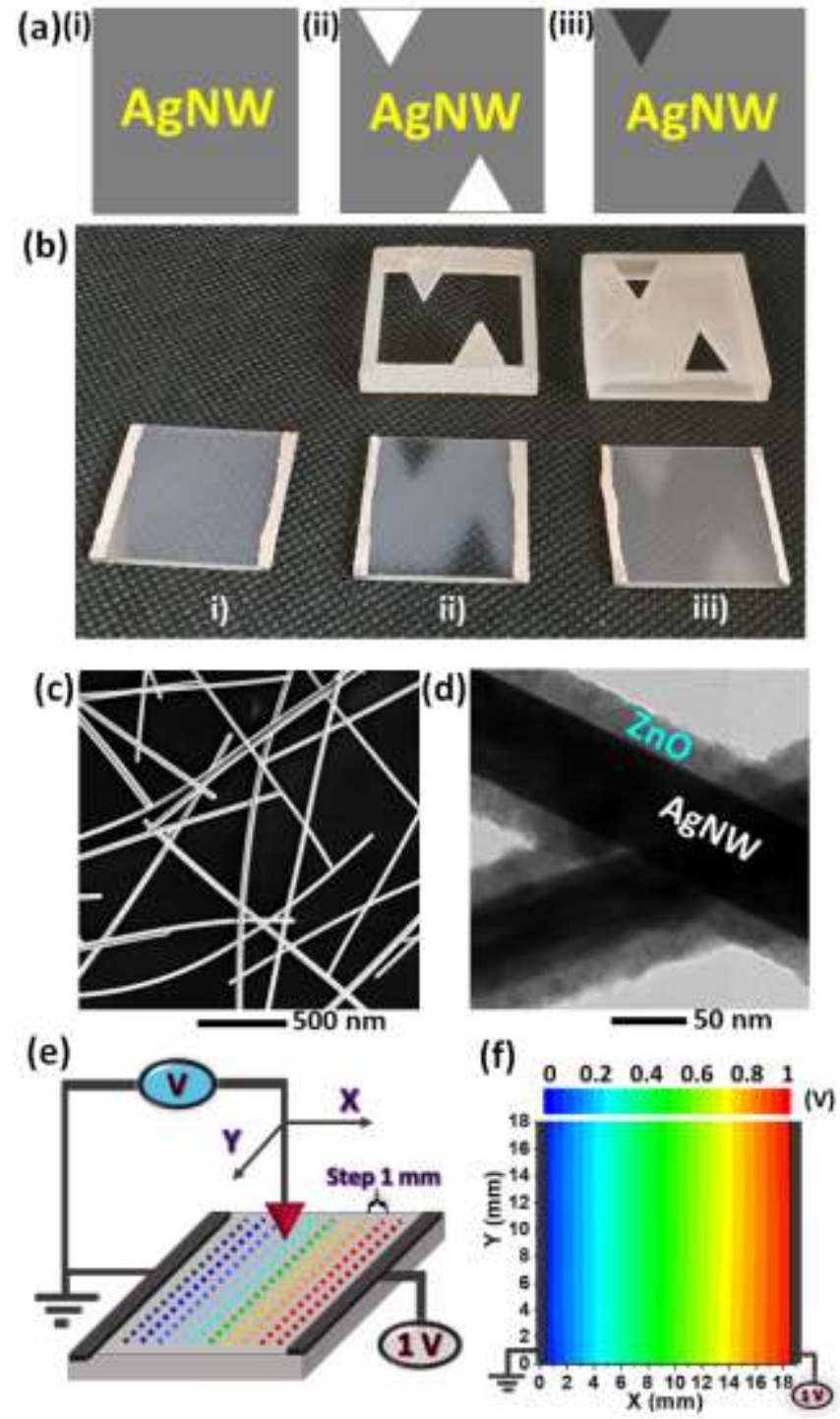

Figure 1 : (a) Schematic representation of the $25 \times 25 \mathrm{~mm}^{2}$ fabricated AgNW networks, for which each grey color shade is associated to the same AgNW network density, with the darker shading indicating the highest one. From the left to the right: i) homogenous (HOM) density on the whole glass substrate, ii) empty triangles (ET) case: specimen without AgNW on the topleft and bottom-right triangles, iii) denser triangles (DT) case: specimen with higher AgNW density in the triangles. (b) Photos of the 3D printed masks used to fabricate the non-homogeneous samples and the three configurations of the studied AgNW networks. (c) SEM image of a bare AgNW network with amd of $125 \mathrm{mg} / \mathrm{m}^{2}$. (d) TEM image of AgNW coated with a conformal thin layer of $30 \pm 5 \mathrm{~nm}$ thick $\mathrm{ZnO}$ deposited by AP-SALD. (e) Schematic representation of the one-probe (1P) mapping: the left silver-paste contact of the specimen is connected to the ground, while a $1 \mathrm{~V}$ bias between opposite electrodes is applied. A tip automatically moves above the studied specimen and touches it point by point to measure the local electric potential. (f) Example of a 1P-map of a homogeneous spray coated AgNW network.

For each type of network geometry, both bare AgNW networks and $\mathrm{AgNW}$ networks coated with a $30 \pm 5 \mathrm{~nm}$ thick $\mathrm{ZnO}$ layer were studied.[54] Figure 1c presents a scanning electron microscopy (SEM) image of a bare AgNW network. The transmission electron microscopy (TEM) image in Figure 
1d shows the conformal $\mathrm{ZnO}$ film coating on the AgNW. Additional SEM and TEM images can be found in Supplementary Information, Figure S2. The six types of specimen (three geometries, with or without the $\mathrm{ZnO}$ coating) exhibit resistance values from 10.2 to $27.6 \Omega$ and an amd of $114 \pm 4 \mathrm{mg} / \mathrm{m}^{2}$, outside the triangular regions. An example of $\mathrm{AgNW} / \mathrm{ZnO}$ with such density is presented in the SEM image of Figure S2b. Figure S3 represents the total and diffuse transmission for a bare and a $\mathrm{ZnO}$ coated $\mathrm{AgNW}$ network. There is a minor influence of the oxide coating on the optical transmittance mainly in the UV range due to the bandgap of $\mathrm{ZnO}$ and the absorption from the $\mathrm{ZnO}$ coated free spaces between $\mathrm{AgNW}$ but the $\mathrm{AgNW} / \mathrm{ZnO}$ maintain very high transmittance. The mean value of total transmittance at 550 nm decreases from $83.3 \%$ to $82.1 \%$, due to the absorption of the coating. Similarly, the coated network becomes slightly more diffuse, with the mean diffuse transmittance increasing from $10.7 \%$ to $11.2 \%$. On the other hand, there is a small increase in the optical transmittance for the coated specimen above the NIR range thanks to the antireflective behavior of the $\mathrm{ZnO}$.[71]

Two main experimental techniques have been used in this work to study the electrical and thermal properties: one-probe (1P) mapping of the electrical potential distribution when the specimen is subjected to a voltage bias and the evolution of electrical resistance during a voltage ramp of $0.5 \mathrm{~V} / \mathrm{min}$ with a simultaneous recording of the surface temperature by IR imaging. The combination of such electrical and thermal analysis contributes to a deeper understanding of failure dynamics. The electrical and thermal properties related to voltage ramp experiments are presented in Table S1. Figure 1e shows a schematic representation of the experimental 1Pmapping set-up, a stepwise spatial measurement of the electrical potential. Figure if presents an example of the resulting map for a $\mathrm{AgNW}$ network, enabling to detect any spatial variations of the electrical potential. IR imagery has already been proved efficient to detect the defects, such as the presence of resistive hotspots and their associated propagation observed during the electrical breakdown of $\mathrm{AgNW}$ networks. [52]

Moreover, simulations of the electric model and Joule heating offer a fair comparison between: i/ the electric potential distribution obtained by the model and the experimental ones measured by 1P-mapping, and ii/ the surface temperature distributions obtained by the model and the IR camera. So far, the majority of the models in the literature related to the $\mathrm{AgNW}$ networks are based on the 2D stick percolation theory to predict the percolation threshold and the macroscopic electrical resistance, and to evaluate the impact of parameters such as the nanowires dimensions and the network density on the network properties.[42,74-76] However, computation limits do not allow the simulations to be made at the same system size as the experimental ones, nor the calculation of the power-induced heating throughout the whole surface of the specimen. In the present work, an approximate thin continuous metallic layer is simulated in order to directly compare the macroscopic electrical and thermal properties with the experimental specimens and the impact of the non-homogeneous deposition of AgNW networks. The simulations were developed with Comsol Multiphysics $®$ and all the details are presented in the Experimental Methods section, complemented with Table S2. For the sake of coherency throughout the article, the same order will be followed in all figures: the first row shows the bare AgNW networks: a) homogeneous (HOM), b) empty triangles (ET) and c) denser triangles (DT); while the second row shows the same geometries for $\mathrm{ZnO}$ coated $\mathrm{AgNW}$ networks: d) homogeneous ( $\mathrm{ZnO}-\mathrm{HOM})$, e) empty triangles ( $\mathrm{ZnO}-\mathrm{ET})$ and f) denser triangles (ZnO-DT). Also, the electrical contacts made of silver-paste, are always placed along opposite vertical sides of the samples.

\section{Bare AgNW networks and the effect of non- homogeneity}

In the first part, we focus on bare AgNW networks through experiments and simulations. 1P-mapping results (Figure 2ac) show that the equipotential lines are predominantly parallel to the vertical electrical contacts for HOM samples, as theoretically expected. Conversely, the tortuosity of the equipotential lines increases for ET and DT samples, reaching a maximum inclination around $30^{\circ}$ to the vertical alignment, close to the empty triangles. Although the empty triangles should appear white, a sporadic presence of nanowires in these regions due to imperfect protection from the mask (see Figure $\mathrm{S} 2 \mathrm{c}$ ) results in blue points inside the triangles. For DT samples, the presence of denser triangles results in smaller deviations from the vertical alignment of $5^{\circ}$ to $10^{\circ}$.

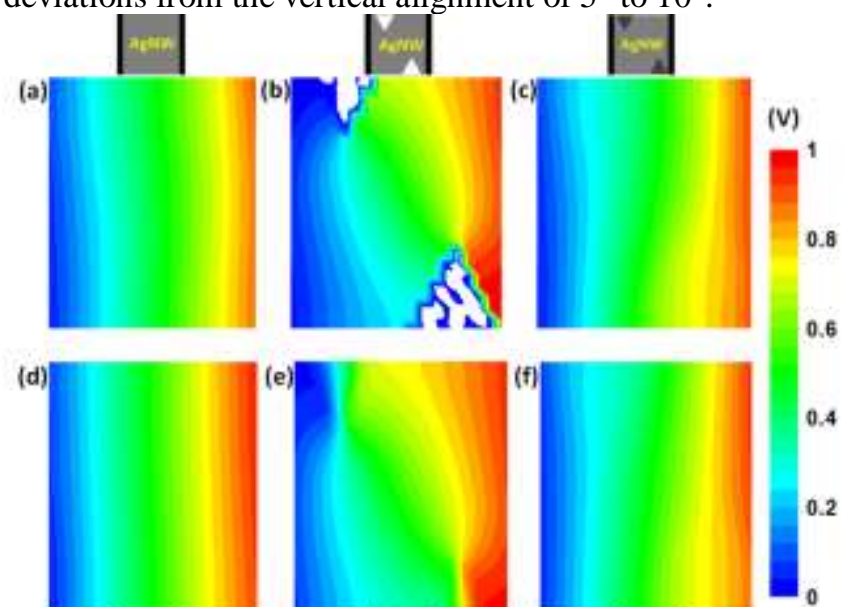

Figure 2: Experimental one-probe electrical maps associated to six types of AgNW networks with $1 \mathrm{~V}$ bias applied between parallel silver-paste vertical electrodes. Bare AgNW networks are plotted on the first row $(a-c)$. The networks coated with $30 \mathrm{~nm}$ thick $\mathrm{ZnO}$ are plotted on the bottom row (d-f). Left column is associated to homogeneous AgNW networks (HOM-a,d), middle column is associated to empty triangles (ET-b,e) and right column to denser triangles (DT-c,f). 
Figure 3 presents the evolution of the electrical resistance of the different specimens submitted to continuously increasing electrical stress. During the voltage ramp, the resistance first increases gradually, but then it follows a sudden sharp increase reaching resistance values in the $M \Omega$ or even $G \Omega$ range within a few seconds, indicating a severe degradation. As previously observed, after this drastic increase the sample returns to the $\mathrm{k} \Omega$ range and cools down.[52,77] For our experiments, we define the voltage failure as the first instant when the time derivative of the resistance is larger than $10^{6} \Omega / \mathrm{sec}$. For the data presented in Figure 3, the HOM AgNW network is stable until higher voltage failure, presenting a well-defined degradation starting at $12.8 \mathrm{~V}$, while the failure voltages for the ET and DT are lower, 11.2 and $11.3 \mathrm{~V}$ respectively.

In Figure 3a-c the evolution of the temperature is also plotted for the HOM-ET-DT bare AgNW cases, as obtained from the IR sequences. The highest temperature values achieved are around $210{ }^{\circ} \mathrm{C}$ for all cases, followed by the cooling down, as Joule heating is negligible when the samples stay at the $\mathrm{G} \Omega$ range. This drop of the temperature is interrupted around $40-50^{\circ} \mathrm{C}$, when the resistance returns to the $\mathrm{k} \Omega$ range, after $200 \mathrm{~s}$. The temperature then exhibits a slight increase and remains around $60^{\circ} \mathrm{C}$ until the end of the voltage ramp. The origin of the electrical drop (Figure 3a-c) after the observed instability is not yet well understood. IR observations show that the local temperature around the formed crack is very high (Figure 3a-c) while voltage is still applied. Therefore one can imagine that electromigration could form again some electrical paths through the crack, leading then to electrical resistance drop.

IR imaging offers interesting information related to the spatial distribution of the temperature, both at the global and the local scales. For the former, prior to degradation, HOM samples tend to present a slightly higher temperature in the center of the sample. The most stressed areas of the nonhomogeneous cases depend on the nature of the heterogeneity: a) along a line between the inside vertices of the triangle for
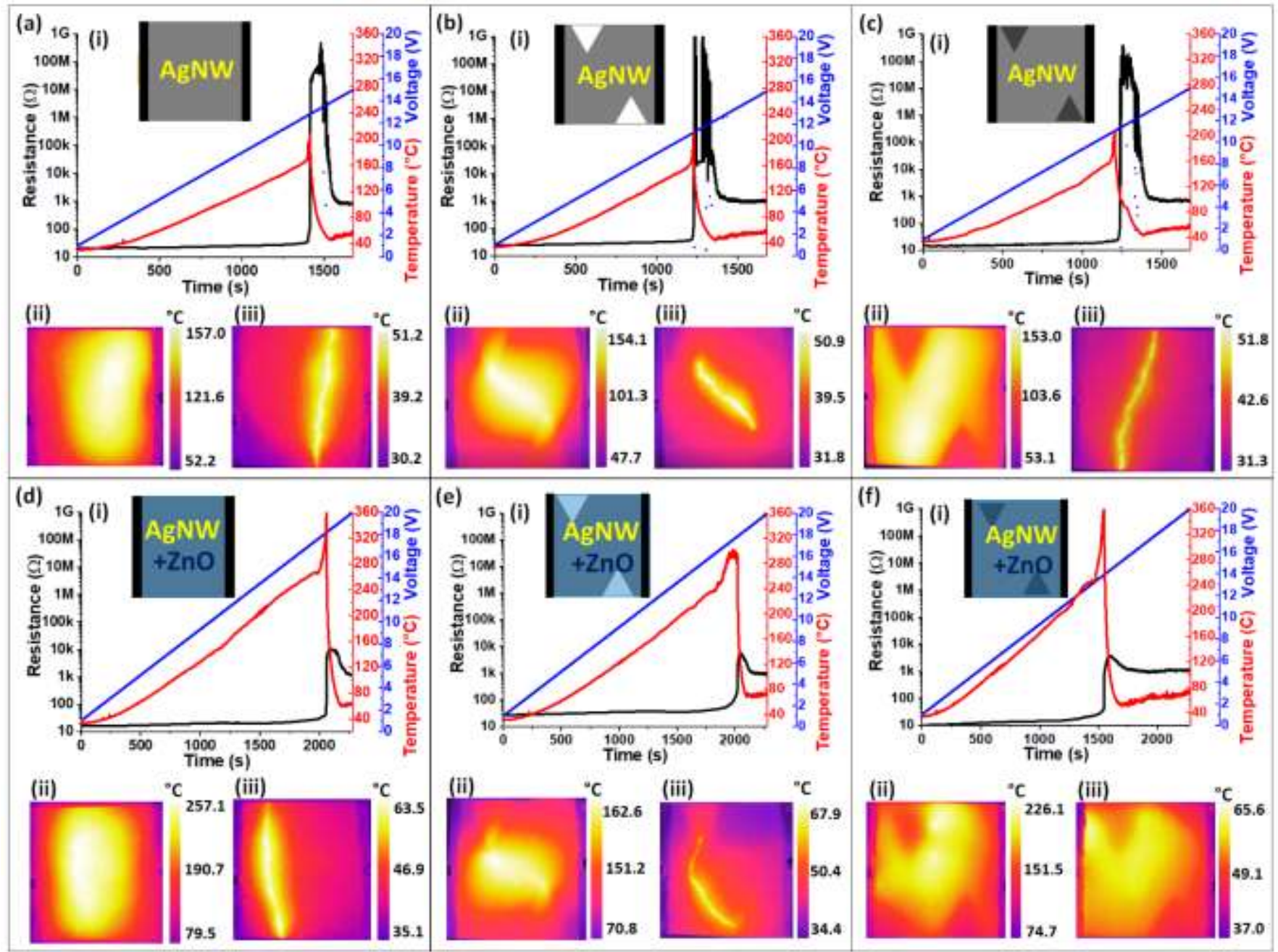

Figure 3: Electrical resistance (black), voltage (blue) and maximum surface temperature (measured from IR imaging and represented in red) during the $0.5 \mathrm{~V} / \mathrm{min}$ voltage ramp applied to: (a-c) bare AgNW networks and (d-f) $30 \mathrm{~nm} \mathrm{ZnO}$ coated AgNW networks. For each network configuration the associated schemas are shown (homogeneous, empty triangles and denser triangles, respectively from left to right). In each case the IR pictures on the left (ii) were taken before the propagation of network failure, while the ones on the right (iii) were taken after the degradation when the resistance had dropped at some $\mathrm{k} \Omega$. The size of all specimens is $25 \times 25 \mathrm{~mm}^{2}$. 
the ET, b) next to the inside edges for DT. The heterogeneity observed in the thermal mappings of DT and ET samples can be easily understood by looking at the 1P-electrical mapping of Figure 2b-c. The denser equipotential lines correspond to the highest temperature, as expected from the Joule effect. The maximum temperature variation between different regions of a given sample is much larger for non-homogeneous samples $\left(60{ }^{\circ} \mathrm{C}\right)$ when compared to the HOM one $\left(45^{\circ} \mathrm{C}\right)$. If this is rather obvious for the sample with less $\mathrm{AgNW}$, it also shows that zones containing a significantly higher amd than the average can also affect the stability of the electrodes.

Concerning more localized effects, IR imaging also shows that some hotspots appear locally along very narrow regions of tens of microns width during the degradation; these same regions also show hotspots after degradation, giving the impression of a thermal crack in the IR sequences (Fig.3 a-iii, b-iii and c-iii). This narrow region may also be revealed through SEM imaging, as shown in Figure 4a. A charging contrast effect helps to localize the damaged nanowires (bright/white nanowires) from the rest of the intact network (dark/black nanowires). This contrast has been recently reported to distinguish the non-conducting AgNW networks by Suemori et al.[78] HOM samples tend to present this crack around the middle of the sample, parallel to the silver paste contacts: this correlates with the temperature measurements and the larger thermal losses on the side contacts compared to the central. The hotspots for the ET sample propagate between the tips of the triangles, while for the DT sample the hotspots are close to the sides of triangles, along the diagonal, at regions where the local electric field is larger, as shown by 1$P$ mapping in Figure 2b and c, as closer equipotential lines are associated to a larger electrical field. Concerning the spatial distribution of temperature after degradation, the crack remains at a higher temperature than the rest of the sample.[52] This can be attributed to the reduced amount of conductive path present in the crack region, causing a local high current density when a voltage is still applied. Therefore, the generated Joule heating is higher than the rest of the

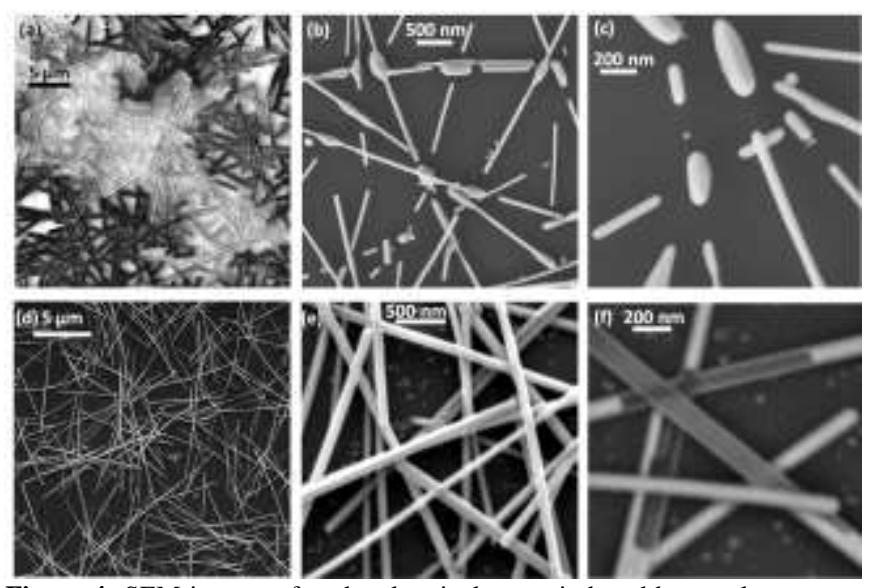

Figure 4: SEM images after the electrical stress induced by a voltage ramp for (a-c) bare AgNW and (d-f) $\mathrm{ZnO}$ coated networks.

sample, which leads to a higher local temperature.

In order to focus on the part of the voltage ramp that corresponds to the electrical degradation, Figure 5 shows the zoom of the time dependence of temperature, resistance, and

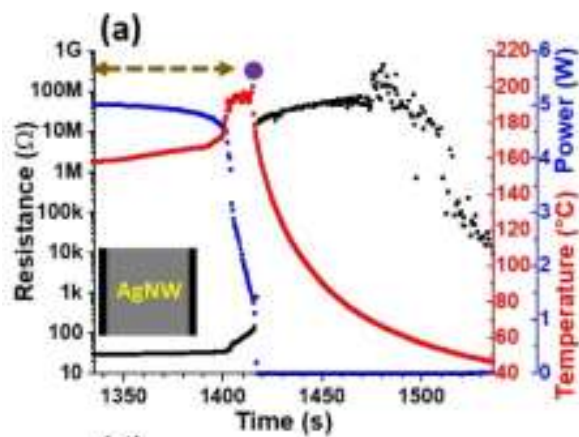

(d)

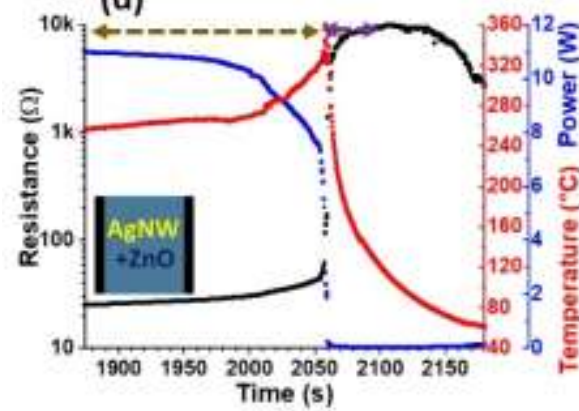

(b)

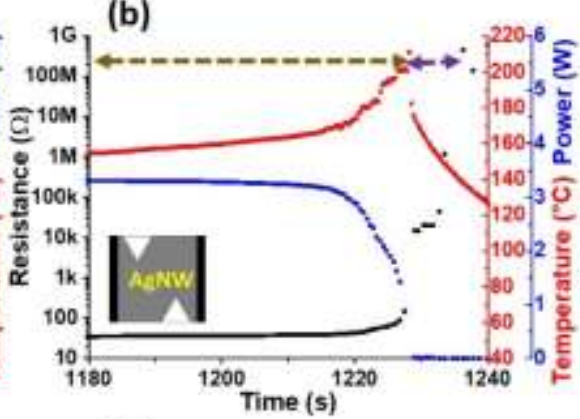

(e)

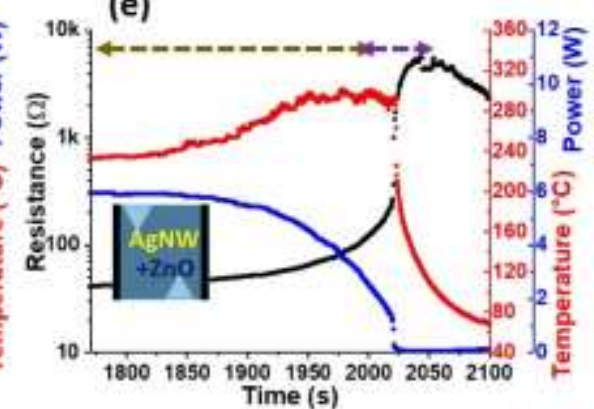

(c)

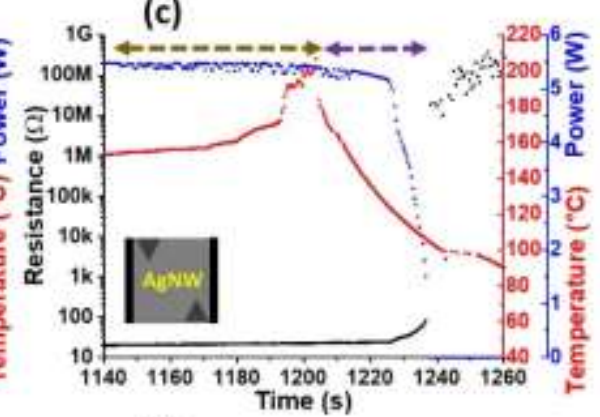

(f)

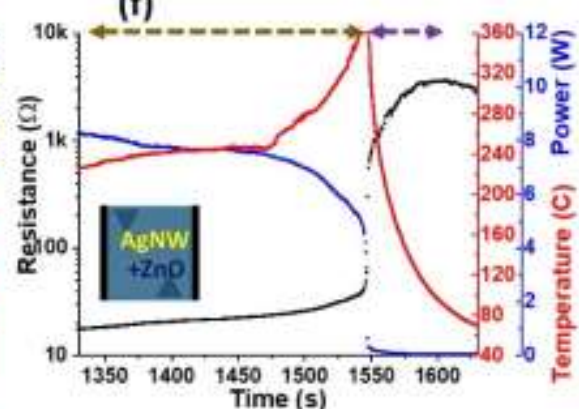

Figure 5: Electrical resistance (black), power (blue) and maximum surface temperature (extracted from IR imaging in red) during a 0.5 V/min voltage ramp applied to: (a-c) bare AgNW networks and (d-f) the $30 \mathrm{~nm} \mathrm{ZnO}$ coated AgNW networks, for the three AgNW network configurations as shown in the inset schemas (homogeneous, empty triangles and denser triangles). For each case, the shown plots start when the power is maximum, while the dashed arrows indicate the duration between (i) maximum power and maximum temperature (ochre/brown line) and (ii) maximum temperature and maximum resistance (purple line). The size of all specimens is $25 \times 25 \mathrm{~mm}^{2}$. 
power in the imminency of the degradation. In this case, we plot the power deduced from the electrical response of the samples, instead of the applied voltage, in order to explore further the mechanisms of failure and to compare the electrical behavior of the different samples. The plots include the power maximum peak and stop when the resistance of each sample returns to $\mathrm{k} \Omega$ range. Throughout the applied voltage ramp, the surface temperature of the AgNW network changes due to the Joule heating. After the first 60-120 s of a slow increase, the temperature starts to increase in a very controlled manner. During this first phase, the resistance increases mildly of about $50 \%$, as compared to the initial value, indicating a safe operation. Then, the temperature increases in a much faster way indicating a degradation process. It is even accompanied by a steep increase of the electrical resistance up to virtually non-conductive values. The dependence of power with time shows that the quick increase of temperature occurs after the maximum of power is reached. This observation combined with the IR sequences suggests that at certain electrical stress, the Joule heating in specific points becomes too large that leads to a local degradation of the network. At the individual wire scale, this indicates that above a certain Joule heating power, the AgNW undergoes a morphological instability. This damage may also be observed macroscopically with the presence of distinctive hotspots. They initiate and propagate to the rest of the sample. This induces macroscopically the increase of the electrical resistance, due to the rupture of the conductive pathways between the electrical contacts. The crack thus indicates electrically degraded areas. The comparison of the position of the hotspots with the electrical distribution reveals a strong influence of the non-homogeneity in the AgNWs density on the failure mechanism observed. Thus, the controlled way of producing samples allowed to disclose the strong correlation between the $1 \mathrm{P}$ mappings (Figure 2a-c) and the IR imaging (Figure 3a-c, ii-IR photos before degradation).

The dynamics of the crack propagation differs for the different density distribution of the bare AgNW networks. Figure 5a-c indicate that the electrical resistance and power exhibit maximum values at different periods (or voltage, see the dashed arrows eye guides) depending on the AgNW network configuration. For instance, $\mathrm{P}_{\max }$ does not occur at the same voltage, thus time, for the three cases: $12.8 \mathrm{~V}$ for HOM, $10.8 \mathrm{~V}$ for ET, and $10.5 \mathrm{~V}$ for DT. The time lag before the temperature reaches its own maximum also depends upon the configuration: $80 \mathrm{~s}, 47 \mathrm{~s}$, and $63 \mathrm{~s}$, for HOM, ET, and DT respectively. The fastest degradation occurs in ET sample, which can be explained mainly by the position of the two hotspots, at the tips of the triangles, leading to large electrical stresses. Also, while for HOM sample the temperature and resistance rise simultaneously, after $\mathrm{P}_{\max }$, in the nonhomogeneous cases the maximum temperature and maximum resistance are clearly separated: $7 \mathrm{~s}$ for ET and $34 \mathrm{~s}$ for DT.
More particularly, in order to explain these time differences, for the ET case, the rise of temperature is followed just a few seconds afterward by a sharp resistance rise, while the hotspots start from the edges of the triangles and the whole middle of the sample heats more. Meanwhile, no current can flow on the top and bottom bands of the network including the empty triangles. The DT case is distinctive because the temperature rises and falls while the power remains in its maximum plateau and the resistance is still increasing. This means that local degradation does not affect the whole sample at the same moment. Indeed, from the beginning of the voltage ramp, the nanowires rich triangle areas heat less than the regions aside them (top right and bottom left). As it has been shown in the IR sequences of Figure 3c, the crack propagates diagonally between these two regions while the bands on the top and bottom remain below $80^{\circ} \mathrm{C}$. The electrical current can still pass through these bands until the thermal crack propagates. As discussed above (Figure 4) the nanowires are degraded in the region where the crack has propagated, while the rest of the sample remains intact. Thus, the crack splits the sample in two separated areas. The degradation of the network is at the local microns-scale, not visible by the naked eye but it is represented by the temperature contrast of the IR images.

Finally, it is important to note that, while the ET and DT samples show different $\mathrm{P}_{\max }$ (3.3 and $5.5 \mathrm{~W}$, respectively) the corresponding Joule heating is the same, with temperatures close to $155^{\circ} \mathrm{C}$. The $\mathrm{P}_{\max }$ value for the ET sample is lower also than HOM networks of similar densities that present a value of $4.8 \pm 0.7 \mathrm{~W}$. In addition, at the moment of $\mathrm{P}_{\max }$, and while the applied voltage is almost the same $(10.5$ and $10.8 \mathrm{~V}$ respectively), the ET sample exhibits a higher resistance (35.5 $\Omega$ ) than the DT one (20.1 $\Omega$ ). Therefore, the ET configuration presents a way to obtain higher local heating for a given power applied, or even a lower one, which can be interesting for $\mathrm{TH}$ applications.

\section{Simulations of electrical and thermal distribution}

Figure 6 reports the simulated spatial distribution of the electrical potential and the current for a $5 \mathrm{~V}$ bias, as well as the comparison with experimental surface temperature data. The initial state at $1 \mathrm{~V}$ is shown in Figure S4. In the HOM case, the electrical equipotential lines are perfectly parallel (Figure 6a). This compares well with the experimental one, as revealed by 1P-electrical mapping (Figure 2a). Additionally, the simulated current density (Figure 6d) has a homogeneous value over the whole studied surface. Figure $6 \mathrm{~g}$ and $\mathrm{j}$ report the calculated and experimental surface temperature. A small but apparent difference between the middle and the edges $(0.2$ ${ }^{\circ} \mathrm{C}$ ) of the sample appears at $1 \mathrm{~V}$ (Figure $\mathrm{S} 4 \mathrm{~g}$ ). The amplitude of such gradient increases, reaching $5{ }^{\circ} \mathrm{C}$ for a voltage of $5 \mathrm{~V}$.

Concerning the non-homogeneous cases, the voltage and current maps are purely derived from the local amount of the conductive material. As reported in Figure $6 \mathrm{~b}$ and c, the 
tortuosity increases, especially in the ET case. As a consequence, the calculated current density varies along the surface of the samples (Figure 6e and f). In ET, the equipotential lines become closer to one another around the tips of the triangles (Figure 6b). The simulation also reveals a maximum value current density value at the tip of the triangles. Low-density areas with sharp angles act as flaws in the structure (Figure 6e). This maximum current value is highly localized and corresponds to the highest temperatures (Figure 6h). The DT case (Figure 6f) shows an intermediate behavior between the homogeneous and ET cases (Figure 6d and e, respectively). The larger amount of AgNWs in the triangle regions increases the maximum current density that can flow on the base of the triangles. These pathways are still available for the current to flow during the degradation, until the moment when the crack arrives and divides the sample in two (Figure 3fiii).

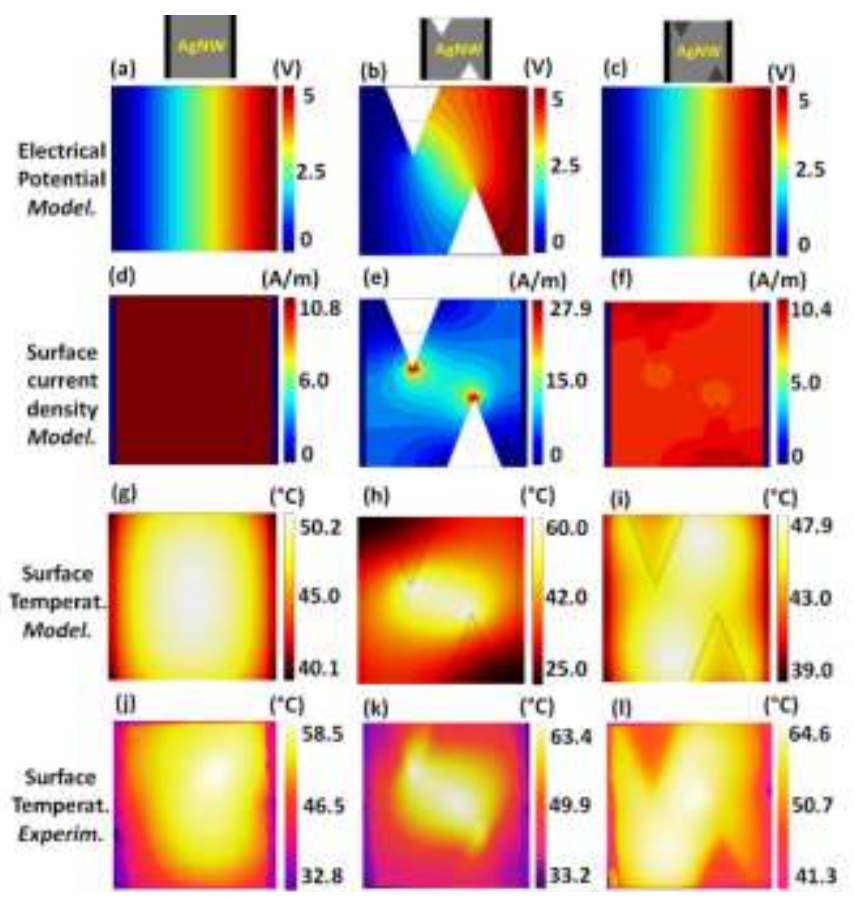

Figure 6: Simulated potential, current density and temperature distributions during electrical stress and comparison with experimental surface temperature for the three specimen geometries. (a-i) Modeling results for the distribution of electrical potential (a-c), surface current density (d-f) and surface temperature distribution ( $\mathrm{g}-\mathrm{i})$, when $5 \mathrm{~V}$ bias is applied between the parallel contacts (left and right vertical sides). (j-1) Experimental results for the spatial distribution of temperature obtained by IR imaging at 5 volts, during a voltage ramp $0.5 \mathrm{~V} / \mathrm{min}$. Three configurations are used (vertical columns): homogeneous layer (HOM-a, d, g, j), networks with empty triangles, (ET-b, $\mathrm{e}, \mathrm{h}, \mathrm{k}$ ) and with more conductive triangles (DT-c, f, i, l). The size of all specimens is $25 \times 25 \mathrm{~mm}^{2}$.

If one compares the two non-homogeneous cases, they show an inverted electrical and thermal behavior; the corresponding mappings of empty and denser triangles seem like complementary stamps the one to the other. However, the dispersion of the current density $\left(\Delta \mathrm{I}_{\text {surface }}\right)$ values is proven to be the significant factor for the local electrical stress and the source of instabilities. It is important to note that not only high values of surface current density or Joule heating are detrimental to the network stability, but also the presence of large gradients show a significant effect. For example, at $5 \mathrm{~V}$ the ET case has 3 times higher $\Delta \mathrm{I}_{\text {surface }}$ than the DT case. For the ET, the current density varies from almost $0 \mathrm{~A} / \mathrm{m}$ close to the contacts to $27.9 \mathrm{~A} / \mathrm{m}$ in the triangle edges. On the other hand, for the DT, the surface current density is $6.5 \mathrm{~A} / \mathrm{m}$ in the triangle edges and its maximum value is over $10 \mathrm{~A} / \mathrm{m}$ at the base of the triangles. This is reflected in the surface temperatures, with the simulations showing a similar trend to the current distribution for the temperature maximum and minimum values too. The temperature difference is around 8 ${ }^{\circ} \mathrm{C}$ for the homogeneous and the DT case, while for the ET is more than 4 times higher, $35^{\circ} \mathrm{C}$, and very localized, as described above.

When comparing the simulated temperature distribution (Figure 6g-i) with the measured one (Figure 6j-1), a very good agreement can be observed, both in terms of temperature ranges and spatial distribution for the three types of electrodes (HOM-ET-DT). Thus, although the temperature dispersion is more significant in the experiments than the simulations, which can be due to the fact that the modeling has taken into account a macroscopic thin film with ideal electrical conduction and thermal losses equilibrium, the simple modeling approach used can predict the electrical and thermal behavior of an entire AgNW network with a fair comparison with experimental observations.

\section{ZnO coating: effect on the electrical distribution and the degradation of AgNW networks}

This last part of the article deals with the effects of $\mathrm{ZnO}$ coating on AgNW degradation. As clearly shown by Figure 3 and Figure 5, a first main difference between coated and bare networks concerns the increase of resistance during degradation: for the $\mathrm{ZnO}$ coated networks the maximum resistance is limited below $10 \mathrm{k} \Omega$, while the resistance of the bare AgNW networks reaches the G $\Omega$ scale. First of all, while the conductivity of the coated electrodes still stems mainly from the nanowires, since the amd value is over 110 $\mathrm{mg} / \mathrm{m}^{2},[64]$ the thin $\mathrm{ZnO}$ layer decreases the electrical resistance, for example from 20.7 to $15.9 \Omega$ for the homogeneous case (Table S1). Since the $\mathrm{ZnO}$ layer covers the whole specimen area, in spite of the low electrical conductivity of the oxide, it enhances the electrical homogeneity. This may be observed with the very parallel equipotential lines for the coated HOM case ( $\mathrm{ZnO}-\mathrm{HOM})$ (Figure 2d) compared to the bare ones (Figure 2a). Similarly, for the coated DT ( $\mathrm{ZnO}-\mathrm{DT}$ ) case, the equipotential lines are less tortuous thanks to the $\mathrm{ZnO}$ layer (Figure $2 \mathrm{c}$ and $\mathrm{f}$ ). The most striking observation after $\mathrm{ZnO}$ coating is for the coated ET (ZnO-ET) configuration, since the "empty" triangles become conductive, thus the electrical potential distribution becomes continuous all over the specimen (Figure $2 b$ and e). 
The increase of overall electrical homogeneity of the AgNW network strongly limits the macroscopic degradation of the whole specimen in terms of electrical resistance and $\mathrm{AgNW}$ morphology impacts. As opposed to the network degradation observed for bare networks (Figure 4a-c), the degradation of coated samples is much smoother since the hotspots are partially short-circuited by the $\mathrm{ZnO}$ thin layer and AgNWs undergo much less damage. This is due to the $\mathrm{ZnO}$ coating prevention of the Ag atom surface diffusion, as proved by the SEM images in Figure 4d-f. While the SALD coating of AgNWs appear very conformal, [54,59] one can suspect that some $\mathrm{AgNW}$ surfaces touching the substrate surface are not coated by the oxide layer. However, this should not have drastic consequences since the surrounding of such faces are coated by the thin oxide layer and then $\mathrm{Ag}$ surface diffusion cannot be active, explaining the stability enhancement after the oxide coating. Although little is known about the role played by the PVP layer during the coating of AgNW by a thin oxide layer, one can observe that eventually both PVP and oxide layers appear to prevent the atomic surface diffusion and therefore enhances the thermal and electrical stability.

Concerning the influence of non-homogeneity in the $\mathrm{ZnO}$ coated AgNW networks, they show the same trend as bare ones, with the $\mathrm{ZnO}$-ET and $\mathrm{ZnO}$-DT degrade at lower voltage compared with $\mathrm{ZnO}-\mathrm{HOM}$ specimen: Figure 3d-f shows that the degradation occurs at $18.2 \mathrm{~V}$ for $\mathrm{ZnO}-\mathrm{HOM}, 17.8 \mathrm{~V}$ for
$\mathrm{ZnO}-\mathrm{ET}$, and $13.9 \mathrm{~V}$ for $\mathrm{ZnO}-\mathrm{DT}$. When comparing these values to the failure of bare $\mathrm{AgNW}$, we observe a significant increase in voltage failure for $\mathrm{ZnO}$ coated samples of all the network configurations. Firstly, for the HOM, this increase is $5.4 \mathrm{~V}$, meaning $42 \%$ which is in agreement to our previous works.[54,64] For the non-homogeneous networks, the ET configuration exhibits the highest benefit from the $\mathrm{ZnO}$ coating, with a $59 \%$ increase of the voltage failure (11.2 V to $17.8 \mathrm{~V}$ for bare and coated $\mathrm{AgNW}$, respectively), while for DT case the increase is $23 \%(11.3 \mathrm{~V}$ to $13.9 \mathrm{~V})$. In summary, the $\mathrm{AgNW} / \mathrm{ZnO}$ composites present a significantly enhanced electrical performance, even for the case of non-homogenous networks. Comparing to several samples measured during our study, the mean voltage failure values for bare $\mathrm{AgNW}$ networks is $(11.5 \pm 1) \mathrm{V}$ and increases to $(17.9 \pm 0.3) \mathrm{V}$ for the $\mathrm{AgNW} / \mathrm{ZnO}$ ones.

Concerning the thermal performance, the composites showed also remarkably higher maximum temperatures before degradation. For the homogeneous and the nonhomogeneous DT, the higher temperature is $360{ }^{\circ} \mathrm{C}$, and for the ET $301.5^{\circ} \mathrm{C}$ at the degradation onset (Figure $5 \mathrm{~d}-\mathrm{f}$ ), while for the bare networks the $\mathrm{T}_{\max }$ are Hom-207 ${ }^{\circ} \mathrm{C}$, ET-211 ${ }^{\circ} \mathrm{C}$, and DT-207 ${ }^{\circ} \mathrm{C}$.

Being functional at higher voltages also means that the maximum power running in the coated samples is increased, i.e. from 5 to $11 \mathrm{~W}$ for the HOM case, from 3.3 to $6 \mathrm{~W}$ for the
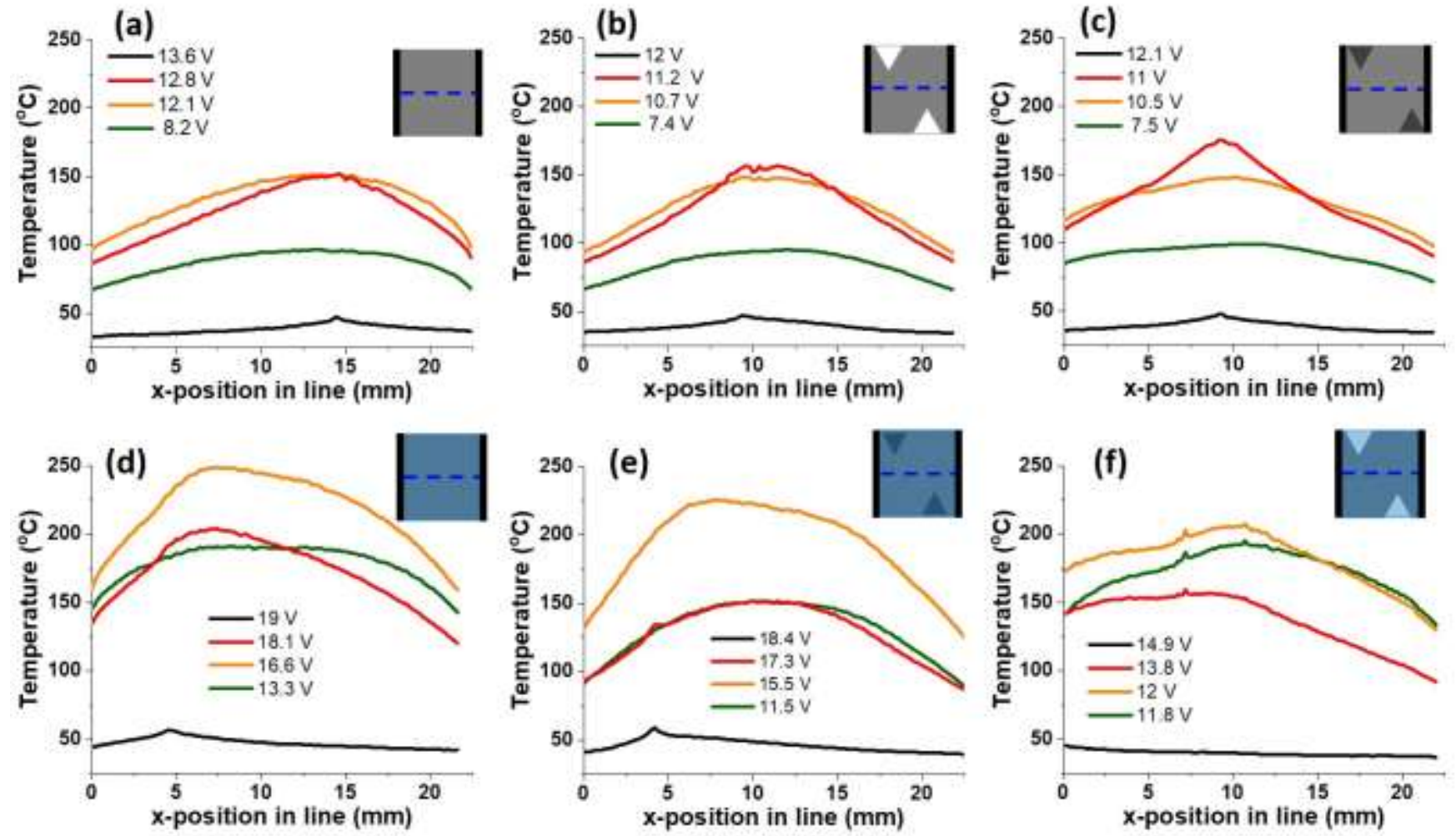

Figure 7: Experimental temperature profiles along a line parallel to the $\mathrm{x}$-axis, in the middle of the samples (see inset dash lines), with the top row (a-c) showing the bare AgNW networks and the bottom row (d-f) the $\mathrm{ZnO}$ coated networks. The temperatures are extracted from the IR sequences during the 0.5 $\mathrm{V} / \mathrm{min}$ voltage ramp. Four different moments of the voltage ramp are chosen each time: far before degradation (green lines), at maximum power (orange lines), at maximum temperature (red lines) and after degradation when the resistance is restored to $\mathrm{k} \Omega$ range (black lines). The applied voltage at each of the four moments is reported in the inset legends. The size of all specimens is $25 \times 25 \mathrm{~mm}^{2}$. 
ET, and from 5.5 to $8.3 \mathrm{~W}$ for the DT. The $\mathrm{ZnO}$ coating decelerates the degradation also in terms of the dynamics of degradation. In addition, the oxide presence, as well as the bare non-homogeneous samples, contributed to a better explanation the dynamics of failure. The time between the maximum power and maximum temperature is now from 2 to 4 times longer than for bare AgNW networks. Even more important is the distinction between the peak of temperature and the peak of resistance, while for the HOM case they happen simultaneously. For the $\mathrm{ZnO}-\mathrm{HOM}$, the dynamics of failure appear different. First of all, the $\mathrm{ZnO}$ coating protects the nanowires from atomic diffusion, so they can be stable at higher Joule heating. Nevertheless, the local electrical stress and thus the Joule heating is so high locally that the degradation still cannot be totally prevented but it is slowed. This local high stress can be the reason why the nonhomogeneous $\mathrm{AgNW} / \mathrm{ZnO}$ networks again degrade faster than the homogeneous coated ones. The coated samples present higher local stress and heating in the same regions as the bare ones, as observed in IR photos before degradation (Figure 3 ii). Secondly, since $\mathrm{ZnO}$ can also electrically conduct current, during the temperature increase it takes more time for the resistive hotspots to propagate in the network. The resistance of the coated electrodes increases in a smoother fashion than the bare networks and it takes longer to reach its highest value. While in HOM samples the temperature rises simultaneously with the resistance (Figure 5a), in the coated homogeneous one it takes $46 \mathrm{~s}$ for the resistance to increase (Figure $5 \mathrm{~d}$ ). This is also the case for the non-homogenous samples, which show a 30-60 s lag (see dashed arrows in the Resistance-PowerTemperature plots in Figure 5).

The third parameter affected by the $\mathrm{ZnO}$ coating is the distribution of heat in the sample during electrical stress. Figure 7 presents the temperature profiles along lines running parallel to the $\mathrm{x}$-axis at the middle of the specimens, as obtained from the IR observations. Four different moments of the voltage ramp are chosen: i/ before degradation (green lines), ii/ when the maximum power runs each sample (orange lines), iii/ when the samples reach the maximum temperature (red lines), and iv/ after degradation when the resistance is restored to $\mathrm{k} \Omega$ range (black lines). To avoid any confusion, it should be noted that the corresponding maximum values of temperature for each sample are not necessarily appearing upon this line of the temperature profiles in the middle of the sample. The temperature profiles for bare samples show that the difference between the middle and the edge points becomes more and more significant as the voltage and maximum temperature increases (Figure $7 \mathrm{a}-\mathrm{c}$ ). This is more prominent for the ET case before the crack propagates (orange line). This is consistent with the high local electrical stress, shown also by experiments and simulations of parts 2.1 and 2.2. In the beginning of the degradation at $11 \mathrm{~V}$, the temperature in the edges is $87^{\circ} \mathrm{C}$ while in the middle is 156
${ }^{\circ} \mathrm{C}$, almost the double. When comparing with the $\mathrm{ZnO}$ coated samples (Figure 7d-f), three differences can be observed. Firstly, the temperature differences between the middle and edges are lower before degradation begins (green lines); the oxide coating contributes to a smoother temperature distribution, which means that can lower down the local thermal stress, where the instability of the AgNW networks originates. Then, the distribution is spatially smoother, without any notable peaks in the middle of the specimen. Finally, the local stress is higher when the power reaches its maximum value (corresponding to the orange lines in Figure 7) and not at the maximum of the temperature (corresponding to the red lines in Figure 7). This can be explained by the dynamics of failure in the $\mathrm{AgNW} / \mathrm{ZnO}$ case, with a decelerated crack propagation and more homogeneous heat dissipation thanks to $\mathrm{ZnO}$. Additionally, the $\mathrm{ZnO}$-DT has a quite different temperature profile. As showed by the IR sequences, the crack, in this case, propagated close to the upper denser triangle and there is also a thin line parallel to the left silver paste contact that showed hotspots during the degradation. This is probably due to the high conformal coating over the nanowires; the high heat finds a way to dissipate close to the edges and at the interface with the contacts. One can observe the same thermal behavior along the diagonal for the bare DT case. These complementary plots are presented in Figure S5, while Table S1 summarizes also all the parameters compared above $\left(\mathrm{R}_{\text {initial }}, \mathrm{V}_{\text {failure }}, \mathrm{P}_{\max }, \mathrm{T}_{\max }\right.$, $I_{\max }$ etc).

\section{Conclusions}

Non-homogeneous networks have been intentionally fabricated using custom 3D printed masks to gain a deeper understanding of the effect of random local fluctuations in the density of AgNW networks on failure, though both experiments and simulations. Such approach appears original since there are very few reports related to the effects of noneven distributions on the electrical or thermal performances. Our results show that local density variations can induce degradation through the development of hotspots in the vicinity of such areas (even for areas in with higher density than the network average density), as a result of current density fluctuations. Furthermore, the temperature range between the hottest and the coolest regions in nonhomogeneous samples is larger than for homogeneous ones. These regions were simulated by simple and macroscopic physical models. In addition, the samples coated with a thin $\mathrm{ZnO}$ layer present a more homogeneous electrical and current distribution, especially for the case of non-homogeneous networks, thanks to the conduction through the oxide, which is most relevant in lower network density regions. As a result, the $\mathrm{ZnO}$ coating increases significantly the stability both for homogeneous and non-homogeneous networks, reaching more than $40 \%$ higher voltage failure and therefore higher 
temperatures, up to $300{ }^{\circ} \mathrm{C}$, while maintaining the superior optical transparency of the electrodes. The results obtained from this work can be applied to other MNW like copper nanowire networks and metallic meshes as well, and contribute to the design of more robust TE with enhanced electrical stability and heating performance for industrial applications such as defrosting/defogging devices or smart windows.

\section{Acknowledgements}

This work was performed within the framework of the Centre of Excellence of Multifunctional Architectured Materials (CEMAM) $\mathrm{n}^{\circ}$ ANR-10-LABX-44-01. Agence Nationale de la Recherche (ANR) is acknowledged for financial support under contracts ANR-18-CE09-0040 (MEANING), ANR-18-CE09-0036 (PANASSE) and ANR15-IDEX-02 (Eco-SESA). The authors would like to warmly thank Laetitia Rapenne for her contribution to the nanowire characterization by TEM and Cesar Arturo Masse de la Huerta for his contribution to the 3D-printing of the masks.

\section{References}

[1] Papanastasiou D T, Schultheiss A, Muñoz-Rojas D, Celle C, Carella A, Simonato J-P and Bellet D 2020 Transparent Heaters: A Review Advanced Functional Materials 1910225

[2] McLellan K, Yoon Y, Leung S N and Ko S H 2020 Recent Progress in Transparent Conductors Based on Nanomaterials: Advancements and Challenges Adv. Mater. Technol. 51900939

[3] Morales-Masis M, De Wolf S, Woods-Robinson R, Ager J W and Ballif C 2017 Transparent Electrodes for Efficient Optoelectronics Advanced Electronic Materials 31600529

[4] Nguyen V H, Gottlieb U, Valla A, Muñoz D, Bellet D and MuñozRojas D 2018 Electron tunneling through grain boundaries in transparent conductive oxides and implications for electrical conductivity: the case of $\mathrm{ZnO}$ :Al thin films Materials Horizons 5 715-26

[5] Giusti G, Consonni V, Puyoo E and Bellet D 2014 High Performance $\mathrm{ZnO}-\mathrm{SnO}_{2}: \mathrm{F}$ Nanocomposite Transparent Electrodes for Energy Applications ACS Appl. Mater. Interfaces 6 14096-107

[6] Tran D-P, Lu H-I and Lin C-K 2018 Conductive Characteristics of Indium Tin Oxide Thin Film on Polymeric Substrate under LongTerm Static Deformation Coatings 8212

[7] Shi J, Shen L, Meng F and Liu Z 2016 Structural, electrical and optical properties of highly crystalline indium tin oxide films fabricated by RPD at room temperature Materials Letters 182 32-5

[8] Barnes T M, Reese M O, Bergeson J D, Larsen B A, Blackburn J L, Beard M C, Bult J and van de Lagemaat J 2012 Comparing the Fundamental Physics and Device Performance of Transparent, Conductive Nanostructured Networks with Conventional Transparent Conducting Oxides Advanced Energy Materials 2353 60

[9] Ma Y and Zhi L 2019 Graphene-Based Transparent Conductive Films: Material Systems, Preparation and Applications Small Methods 31800199

[10] Yan W, Page A, Nguyen-Dang T, Qu Y, Sordo F, Wei L and Sorin F 2019 Advanced Multimaterial Electronic and Optoelectronic Fibers and Textiles Adv. Mater. 311802348

[11] Zhou Y and Azumi R 2016 Carbon nanotube based transparent conductive films: progress, challenges, and perspectives Sci Technol Adv Mater 17 493-516

[12] Xu Y and Liu J 2016 Graphene as Transparent Electrodes: Fabrication and New Emerging Applications Small 12 1400-19
[13] Gueye M N, Carella A, Demadrille R and Simonato J-P 2017 AllPolymeric Flexible Transparent Heaters ACS Appl. Mater. Interfaces $927250-6$

[14] Khan A, Lee S, Jang T, Xiong Z, Zhang C, Tang J, Guo L J and Li W-D 2016 High-Performance Flexible Transparent Electrode with an Embedded Metal Mesh Fabricated by Cost-Effective Solution Process Small 12 3021-30

[15] Bellet D, Lagrange M, Sannicolo T, Aghazadehchors S, Nguyen V H, Langley D P, Muñoz-Rojas D, Jiménez C, Bréchet Y and Nguyen N D 2017 Transparent Electrodes Based on Silver Nanowire Networks: From Physical Considerations towards Device Integration Materials 10570

[16] Li X, Wang Y, Yin C and Yin Z 2020 Copper nanowires in recent electronic applications: progress and perspectives J. Mater. Chem. C 8 849-72

[17] Shah K W and Xiong T 2019 Multifunctional Metallic Nanowires in Advanced Building Applications Materials 121731

[18] Sannicolo T, Lagrange M, Cabos A, Celle C, Simonato J-P and Bellet D 2016 Metallic Nanowire-Based Transparent Electrodes for Next Generation Flexible Devices: a Review Small 12 6052-75

[19] Azani M, Hassanpour A and Torres T 2020 Benefits, Problems, and Solutions of Silver Nanowire Transparent Conductive Electrodes in Indium Tin Oxide (ITO)-Free Flexible Solar Cells Adv. Energy Mater. 2002536

[20] Lagrange M, Langley D P, Giusti G, Jiménez C, Bréchet Y and Bellet D 2015 Optimization of Silver Nanowire-Based Transparent Electrodes: Effects of Density, Size and Thermal Annealing Nanoscale 7 17410-23

[21] Madeira A, Papanastasiou D T, Toupance T, Servant L, TréguerDelapierre M, Bellet D and Goldthorpe I A 2020 Rapid synthesis of ultra-long silver nanowires for high performance transparent electrodes Nanoscale Adv. 2 3804-8

[22] Kim D-J, Shin H-I, Ko E-H, Kim K-H, Kim T-W and Kim H-K 2016 Roll-to-roll slot-die coating of $400 \mathrm{~mm}$ wide, flexible, transparent $\mathrm{Ag}$ nanowire films for flexible touch screen panels $S c i$ Rep 634322

[23] Kang H, Song S-J, Sul Y E, An B-S, Yin Z, Choi Y, Pu L, Yang CW, Kim Y S, Cho S M, Kim J-G and Cho J H 2018 EpitaxialGrowth-Induced Junction Welding of Silver Nanowire Network Electrodes ACS Nano 12 4894-902

[24] Xu X, Han G, Yu H, Jin X, Yang J, Lin J and Ma C 2020 Resistance change of stretchable composites based on inkjet-printed silver nanowires J. Phys. D: Appl. Phys. 53 05LT02

[25] Wang Y, Zhang L and Wang D 2019 Ultrastretchable Hybrid Electrodes of Silver Nanowires and Multiwalled Carbon Nanotubes Realized by Capillary-Force-Induced Welding Adv. Mater. Technol. 41900721

[26] Kim Y and Kim J-W 2016 Silver nanowire networks embedded in urethane acrylate for flexible capacitive touch sensor Applied Surface Science 363 1-6

[27] Sohn H, Park C, Oh J-M, Kang S W and Kim M-J 2019 Silver Nanowire Networks: Mechano-Electric Properties and Applications Materials 122526

[28] Singh M and Rana S 2020 Silver and copper nanowire films as costeffective and robust transparent electrode in energy harvesting through photovoltaic: A review Materials Today Communications 24101317

[29] Liu J, Jia D, Gardner J M, Johansson E M J and Zhang X 2019 Metal nanowire networks: Recent advances and challenges for new generation photovoltaics Materials Today Energy 13 152-85

[30] Jung E, Kim C, Kim M, Chae H, Cho J H and Cho S M 2017 Rollto-roll preparation of silver-nanowire transparent electrode and its application to large-area organic light-emitting diodes Organic Electronics 41 190-7

[31] Park H-G, Heo G-S, Park S-G, Jeong H-C, Lee J H and Seo D-S 2015 Silver Nanowire Networks as Transparent Conducting Films for Liquid Crystal Displays ECS Solid State Lett. 4 R50-2

[32] Celle C, Mayousse C, Moreau E, Basti H, Carella A and Simonato J-P 2012 Highly flexible transparent film heaters based on random networks of silver nanowires Nano Research 5 427-33

[33] Huang Y, Tian Y, Hang C, Liu Y, Wang S, Qi M, Zhang H and Zhao J 2019 Self-Limited Nanosoldering of Silver Nanowires for 
High-Performance Flexible Transparent Heaters ACS Applied Materials \& Interfaces $1121850-8$

[34] Min K, Umar M, Seo H, Yim J H, Kam D G, Jeon H, Lee S and Kim S 2017 Biocompatible, optically transparent, patterned, and flexible electrodes and radio-frequency antennas prepared from silk protein and silver nanowire networks RSC Advances 7 574-80

[35] Zeng Z, Wu T, Han D, Ren Q, Siqueira G and Nyström G 2020 Ultralight, Flexible, and Biomimetic Nanocellulose/Silver Nanowire Aerogels for Electromagnetic Interference Shielding ACS Nano 14 2927-38

[36] Chen W, Liu L-X, Zhang H-B and Yu Z-Z 2020 Flexible, Transparent, and Conductive $\mathrm{Ti}_{3} \mathrm{C}_{2} \mathrm{~T}{ }_{x}$ MXene-Silver Nanowire Films with Smart Acoustic Sensitivity for High-Performance Electromagnetic Interference Shielding ACS Nano 14 16643-53

[37] Lu S, Cardenas J A, Worsley R, Williams N X, Andrews J B, Casiraghi C and Franklin A D 2019 Flexible, Print-in-Place 1D-2D Thin-Film Transistors Using Aerosol Jet Printing ACS Nano 13 11263-72

[38] Yuksel R, Coskun S, Kalay Y E and Unalan H E 2016 Flexible, silver nanowire network nickel hydroxide core-shell electrodes for supercapacitors Journal of Power Sources 328 167-73

[39] Guan F, Xie Y, Wu H, Meng Y, Shi Y, Gao M, Zhang Z, Chen S, Chen Y, Wang H and Pei Q 2020 Silver Nanowire-Bacterial Cellulose Composite Fiber-Based Sensor for Highly Sensitive Detection of Pressure and Proximity ACS Nano 14 15428-39

[40] Huang W-R, He Z, Wang J-L, Liu J-W and Yu S-H 2019 Mass Production of Nanowire-Nylon Flexible Transparent Smart Windows for PM2.5 Capture iScience 12 333-41

[41] Fantanas D, Brunton A, Henley S J and Dorey R A 2018 Investigation of the mechanism for current induced network failure for spray deposited silver nanowires Nanotechnology 29465705

[42] Khaligh H H, Xu L, Khosropour A, Madeira A, Romano M, Pradére C, Tréguer-Delapierre M, Servant L, Pope M A and Goldthorpe I A 2017 The Joule heating problem in silver nanowire transparent electrodes Nanotechnology $\mathbf{2 8} 425703$

[43] Khaligh H H and Goldthorpe I A 2013 Failure of silver nanowire transparent electrodes under current flow Nanoscale research letters $81-6$

[44] Chen D, Zhao F, Tong K, Saldanha G, Liu C and Pei Q 2016 Mitigation of Electrical Failure of Silver Nanowires under Current Flow and the Application for Long Lifetime Organic Light-Emitting Diodes Advanced Electronic Materials 21600167

[45] Liu C, Ainsworth C A, Sampson W W and Derby B 2020 Fatigue and the electrical resistance of silver nanowire networks Scripta Materialia 181 97-100

[46] Mayousse C, Celle C, Fraczkiewicz A and Simonato J-P 2015 Stability of silver nanowire based electrodes under environmental and electrical stresses Nanoscale 7 2107-15

[47] Schrenker N J, Xie Z, Schweizer P, Moninger M, Werner F, Karpstein N, Mačković M, Spyropoulos G D, Göbelt M, Christiansen S, Brabec C J, Bitzek E and Spiecker E 2021 Microscopic Deformation Modes and Impact of Network Anisotropy on the Mechanical and Electrical Performance of Five-fold Twinned Silver Nanowire Electrodes ACS Nano 15 362-76

[48] Batra N M, Syed A and Costa P M F J 2019 Current-induced restructuring in bent silver nanowires Nanoscale 11 3606-18

[49] Maize K, Das S R, Sadeque S, Mohammed A M S, Shakouri A, Janes D B and Alam M A 2015 Super-Joule heating in graphene and silver nanowire network Applied Physics Letters 106143104

[50] Lagrange M, Sannicolo T, Muñoz-Rojas D, Lohan B G, Khan A, Anikin M, Jiménez C, Bruckert F, Bréchet Y, and D Bellet 2017 Understanding the mechanisms leading to failure in metallic nanowire-based transparent heaters, and solution for stability enhancement Nanotechnology 28055709

[51] Kholid F N, Huang H, Zhang Y and Fan H J 2016 Multiple electrical breakdowns and electrical annealing using high current approximating breakdown current of silver nanowire network Nanotechnology 27025703

[52] Sannicolo T, Charvin N, Flandin L, Kraus S, Papanastasiou D T, Celle C, Simonato J-P, Muñoz-Rojas D, Jiménez C and Bellet D 2018 Electrical Mapping of Silver Nanowire Networks: A Versatile
Tool for Imaging Network Homogeneity and Degradation Dynamics during Failure ACS Nano 12 4648-59

[53] Zhu Y, Wan T, Guan P, Wang Y, Wu T, Han Z, Tang G and Chu D 2020 Improving thermal and electrical stability of silver nanowire network electrodes through integrating graphene oxide intermediate layers Journal of Colloid and Interface Science 566 375-82

[54] Khan A, Nguyen V H, Muñoz-Rojas D, Aghazadehchors S, Jiménez C, Nguyen N D and Bellet D 2018 Stability Enhancement of Silver Nanowire Networks with Conformal ZnO Coatings Deposited by Atmospheric Pressure Spatial Atomic Layer Deposition ACS Applied Materials \& Interfaces $1019208-17$

[55] Chae W H, Sannicolo T and Grossman J C 2020 Double-Sided Graphene Oxide Encapsulated Silver Nanowire Transparent Electrode with Improved Chemical and Electrical Stability ACS Appl. Mater. Interfaces 12 17909-20

[56] Pham A-T, Nguyen X-Q, Tran D-H, Ngoc Phan V, Duong T-T and Nguyen D-C 2016 Enhancement of the electrical properties of silver nanowire transparent conductive electrodes by atomic layer deposition coating with zinc oxide Nanotechnology 27335202

[57] Go M, Hwang B and Lim S 2019 Highly reliable mulberry paper (Hanji)-based electrode with printed silver nanowire/zinc oxide hybrid for soft electronics Materials and Manufacturing Processes 34 1605-11

[58] Lee J-M, Kim Y-H, Kim H-K, Kim H-J and Hong C-H 2020 Effect of AlOx protection layer on $\mathrm{AgNW}$ s for flexible transparent heater Sci Rep $\mathbf{1 0} 4592$

[59] Aghazadehchors S, Nguyen V H, Muñoz-Rojas D, Jiménez C, Rapenne L, Nguyen N D and Bellet D 2019 Versatility of bilayer metal oxide coatings on silver nanowire networks for enhanced stability with minimal transparency loss Nanoscale 11 19969-79

[60] Celle C, Cabos A, Fontecave T, Laguitton B, Benayad A, Guettaz L, Pélissier N, Nguyen V H, Bellet D, Muñoz-Rojas D and Simonato J-P 2018 Oxidation of copper nanowire based transparent electrodes in ambient conditions and their stabilization by encapsulation: application to transparent film heaters Nanotechnology 29085701

[61] Hwang B, An Y, Lee H, Lee E, Becker S, Kim Y-H and Kim H 2017 Highly Flexible and Transparent Ag Nanowire Electrode Encapsulated with Ultra-Thin Al2O3: Thermal, Ambient, and Mechanical Stabilities Sci Rep 741336

[62] Göbelt M, Keding R, Schmitt S W, Hoffmann B, Jäckle S, Latzel M, Radmilović V V, Radmilović V R, Spiecker E and Christiansen S 2015 Encapsulation of silver nanowire networks by atomic layer deposition for indium-free transparent electrodes Nano Energy 16 196-206

[63] Cheong H-G, Kim J-H, Song J-H, Jeong U and Park J-W 2015 Highly flexible transparent thin film heaters based on silver nanowires and aluminum zinc oxides Thin Solid Films $589633-41$

[64] Nguyen V H, Resende J, Papanastasiou D T, Fontanals N, Jiménez C, Muñoz-Rojas D and Bellet D 2019 Low-cost fabrication of flexible transparent electrodes based on $\mathrm{Al}$ doped $\mathrm{ZnO}$ and silver nanowire nanocomposites: impact of the network density Nanoscale 11 12097-107

[65] Sannicolo T, Chae W H, Mwaura J, Bulović V and Grossman J C 2021 Silver Nanowire Back Electrode Stabilized with Graphene Oxide Encapsulation for Inverted Semitransparent Organic Solar Cells with Longer Lifetime ACS Appl. Energy Mater. 4 1431-41

[66] Barquinha P 2012 Transparent oxide electronics: from materials to devices (Chichester: Wiley)

[67] Chang I, Lee J, Lee Y, Lee Y H, Ko S H and Cha S W 2017 Thermally stable Ag@ZrO 2 core-shell via atomic layer deposition Materials Letters 188 372-4

[68] Huang Y, Tian Y, Hang C, Liu Y, Wang S, Qi M, Zhang H and Peng Q $2019 \mathrm{TiO}_{2}$-Coated Core-Shell Ag Nanowire Networks for Robust and Washable Flexible Transparent Electrodes ACS Appl. Nano Mater. 2 2456-66

[69] Milano G, Cultrera A, Bejtka K, De Leo N, Callegaro L, Ricciardi C and Boarino L 2020 Mapping Time-Dependent Conductivity of Metallic Nanowire Networks by Electrical Resistance Tomography toward Transparent Conductive Materials ACS Appl. Nano Mater. 3 11987-97

[70] Muñoz-Rojas D, Nguyen V H, Masse de la Huerta C, Aghazadehchors S, Jiménez C and Bellet D 2017 Spatial Atomic 
Layer Deposition (SALD), an emerging tool for energy materials. Application to new-generation photovoltaic devices and transparent conductive materials Comptes Rendus Physique 18 391-400

[71] Nguyen V H, Resende J, Jiménez C, Deschanvres J-L, Carroy P, Muñoz D, Bellet D and Muñoz-Rojas D 2017 Deposition of ZnO based thin films by atmospheric pressure spatial atomic layer deposition for application in solar cells Journal of Renewable and Sustainable Energy 9021203

[72] Mayousse C, Celle C, Moreau E, Mainguet J-F, Carella A and Simonato J-P 2013 Improvements in Purification of Silver Nanowires by Decantation and Fabrication of Flexible Transparent Electrodes. Application to Capacitive Touch Sensors Nanotechnology $\mathbf{2 4} 215501$

[73] Sorel S, Bellet D and Coleman J N 2014 Relationship between Material Properties and Transparent Heater Performance for Both Bulk-like and Percolative Nanostructured Networks ACS Nano 8 4805-14

[74] Benda R, Cancès E and Lebental B 2019 Effective resistance of random percolating networks of stick nanowires: Functional dependence on elementary physical parameters Journal of Applied Physics 126044306

[75] Langley D P, Lagrange M, Nguyen N D and Bellet D 2018 Percolation in networks of 1-dimensional objects: comparison between Monte Carlo simulations and experimental observations Nanoscale Horizons 3 545-50

[76] Mutiso R M, Sherrott M C, Rathmell A R, Wiley B J and Winey K I 2013 Integrating Simulations and Experiments To Predict Sheet Resistance and Optical Transmittance in Nanowire Films for Transparent Conductors ACS Nano 7 7654-63

[77] Charvin N, Resende J, Papanastasiou D T, Muñoz-Rojas D, Jiménez C, Nourdine A, Bellet D and Flandin L 2021 Dynamic degradation of metallic nanowire networks under electrical stress: a comparison between experiments and simulations Nanoscale Adv. $3675-81$

[78] Suemori K, Watanabe Y, Fukuda N and Uemura S 2020 Voltage Contrast in Scanning Electron Microscopy to Distinguish Conducting Ag Nanowire Networks from Nonconducting Ag Nanowire Networks ACS Omega 5 12692-7 